\title{
The Kervaire invariant and surgery theory
}

\author{
Edgar H. Brown, Jr.
}

\begin{abstract}
We give an expository account of the development of the Kervaire invariant and its generalizations with emphasis on its applications to surgery and, in particular, to the existence of stably parallelizable manifolds with Kervaire invariant one.
\end{abstract}

\section{INTRODUCTION}

As an expository device we describe the development of this subject in chronological order beginning with Kervaire's original paper ([10]) and Kervaire-Milnor's Groups of Homotopy Spheres ([11]) followed by Frank Peterson's and my work using Spin Cobordism ([5], [7]), Browder's application of the Adams spectral sequence to the Kervaire invariant one problem ([3]), Browder-Novikov surgery ([16]) and finally an overall generalization of mine $([6])$. In a final section we describe, with no detail, other work and references for these areas. We do not give any serious proofs until we get to the "overall generalization" sections where we prove the results about the generalized Kervaire invariant and Browder's Kervaire invariant one results.

\section{Cobordism Preliminaries.}

We make $\mathbb{R}^{N} \subset \mathbb{R}^{N+1}$ by identifying $x \in \mathbb{R}^{N}$ with $(x, 0)$. Then $B O_{k}=$ $\bigcup G_{k, l}$, where $G_{k, l}$ is the space of $k$ dimensional linear subspaces of $\mathbb{R}^{k+l}$ and the universal bundle $\zeta_{k} \rightarrow B O_{k}$ is the space of all pairs $(P, v)$, where $v \in P \in G_{k, l}$ for some $l$. A vector bundle is assumed to have a metric on its fibres. Hence if $\xi$ is a $k$-plane bundle over $X$, it has associated disc and sphere bundles, $D \xi$ and $S \xi$, a Thom space $T \xi=D \xi / S \xi$, and a Thom class $U_{k} \in H^{k}(T \xi)$ (coefficients $\mathbb{Z}$ or $\mathbb{Z} / 2 \mathbb{Z}$ as appropriate).

Throughout this paper " $m$-manifold" means a smooth, compact manifold of dimension $m$, equipped with a smooth embedding into Euclidean space, $\mathbb{R}^{m+k}, k$ large $(k>2 m+1)$. If $M$ is such a manifold, its tangent 
and normal bundles are given by

$$
\begin{aligned}
& \tau(M)=\left\{(x, v) \in M \times \mathbb{R}^{m+k} \mid v \text { is tangent to } M \text { at } x\right\}, \\
& \nu(M)=\left\{(x, v) \in M \times \mathbb{R}^{m+k} \mid v \text { is perpendicular to } M \text { at } x\right\} .
\end{aligned}
$$

One associates to $M$ a map $t: S^{m+k}=\mathbb{R}^{m+k} \cup\{\infty\} \longrightarrow T(\nu)$, the Thom construction, as follows: For $\epsilon>0$ sufficiently small, e $: D_{\epsilon}(\nu(M)) \rightarrow$ $\mathbb{R}^{m+k}$, given by $e(x, v)=x+v$, is an embedding. Let $t(u)=(x, v / \epsilon)$ if $u=e(x, v)$ and $=\{S(\nu(M))\}$ otherwise. If $\xi$ is a $k$-plane bundle over $X$, a $\xi$-structure on a manifold $M$ is a bundle map $f: \nu(M) \longrightarrow \xi ; f_{M}: M \longrightarrow X$ denotes the underlying map.

We define the $m^{\text {th }} \xi$-cobordism group, $\Omega_{m}(\xi)$, to be the set of pairs $(M, f)$, where $M$ is a closed $m$-manifold with a $\xi$-structure $f$, modulo the equivalence relation generated by the following to relations. If $i: M \subset$ $\mathbb{R}^{m+k}$ and $j: \mathbb{R}^{m+k} \subset \mathbb{R}^{m+k+1}$ are their given inclusions, then $M$ equipped with $i$ is equivalent to $M$ equipped with $j i$. Also $\left(M_{1}, f_{1}\right)$ and $\left(M_{2}, f_{2}\right)$ are equivalent if they are $\xi$-cobordant, that is, there is a $(m+1)$-manifold $N$ with a $\xi$-structure $F$ and an embedding $N \subset \mathbb{R}^{m+k} \times[0,1]$ such that $\partial N=M_{1} \cup M_{2}, N$ is perpendicular to $\mathbb{R}^{m+k} \times\{0,1\},(N, F) \cap \mathbb{R}^{m+k} \times\{i-$ $1\}=\left(M_{i}, f_{i}\right)$. Disjoint union of pairs $\left(M_{1}, f_{1}\right)$ and $\left(M_{2}, f_{2}\right)$ makes $\Omega_{m}(\xi)$ into an abelian group.

Theorem 2.1 (Thom [21]). Sending $(M, f)$ to $T(f) t: S^{m+k} \longrightarrow T(\xi)$ induces an isomorphism, $\Psi: \Omega_{m}(\xi) \longrightarrow \pi_{m+k}(T(\xi))$.

Sometimes when $\xi$ is a bundle over a particular $X$, we denote $T \xi$ by $T X$ and $\Omega_{m}(\xi)$ by $\Omega_{m}(X)$, or when $X=B G_{k}$ by $\Omega_{m}(G)$.

\section{Groups of Homotopy Spheres}

Kervaire and Milnor ([11]) defined the group of homotopy $m$-spheres, $\theta_{m}$, to be the set of closed, oriented $m$-manifolds homotopy equivalent to $S^{m}$ (for $m>4$, by Smale's Theorem, homeomorphic to $S^{m}$ ) modulo the relation of $h$-cobordism (for a cobordism $N$ between $M_{1}$ and $M_{2}$, the inclusions of $M_{i}$ into $N$ are required to be homotopy equivalences). Addition is defined using the connected sum operation. Using Bott's computation of $\pi_{*}(B O)$ and results of Adams concerning the $J$-homomorphism, they prove:

Theorem 3.1. If $M$ is a homotopy $m$-sphere, $\nu(M)$ is trivial ( $k$ large).

For the remainder of this section we assume $m>4$. Let $0^{k}$ denote the vector bundle $\mathbb{R}^{k} \longrightarrow p t$. Thus an $0^{k}$-structure on $M$ is a framing of $\nu(M)$. If $M$ is a homotopy $m$-sphere, choosing a framing of $\nu(M)$ gives an 
element of $\Omega_{m}\left(0^{k}\right) \approx \pi_{m+k}\left(S_{k}\right)$ and a simple argument shows that changing the framing adds to this element an element in the image of $J$. Thus we have an induced map $\Psi: \theta_{m} \longrightarrow \pi_{m+k}\left(S_{k}\right) / \operatorname{Im} J$. The Kervaire-Milnor paper is mainly devoted to computing the kernel and cokernel of this map, both of which turn out to be finite cyclic groups; the kernel is denoted by $b P_{m+1}$ $=$ homotopy spheres bounded by stably parallelizable $(m+1)$-manifolds.

\section{Surgery}

The process of doing surgery on an $m$-manifold with respect to an embedding $g: S^{n} \times D^{m-n} \longrightarrow M\left(S^{q-1}\right.$ and $D^{q}$ are the unit sphere and disc in $\mathbb{R}^{q}$ ) consists of producing a new manifold $M^{\prime}$ and a cobordism $N$ between $M$ and $M^{\prime}$ as follows. Let $N$ be a smoothed version of the identification space formed from $M \times I \cup D^{n+1} \times D^{m-n}$ by identifying $(x, y) \in$ $S^{n} \times D^{m-n}$ with $(g(x, y), 1)$. The boundary of $N$ consists of three parts, $M=M \times\{0\}, M-g\left(S^{n} \times D^{m-n}\right) \cup D^{n+1} \times S^{m-n-1}$ and $(\partial M) \times I$. In what follows $M$ is closed or its boundary is a homotopy $(m-1)$-sphere which we can cone off to form a topological closed manifold. Henceforth we ignore $\partial M$. Note $M^{\prime}$ has an embedding $D^{n+1} \times S^{m-n-1} \longrightarrow M^{\prime}$, namely the inclusion, and applying surgery to it gives $M$. We define a $\xi$-surgery to be one in which $M, M^{\prime}$ and $N$ have $\xi$-structures making a $\xi$-cobordism. For our applications to $b P_{m+1}$ we use $\xi=0^{k}$. The change in homology going from $M$ to $M^{\prime}$ can be easily computed from the homology exact sequences of the pairs $(N, M)$ and $\left(N, M^{\prime}\right)$ and the observation that $H_{q}(N, M) \approx H_{q}\left(D^{n+1}, S^{n}\right)$. If $2 n<m-1, H_{q}(M) \approx H_{q}\left(M^{\prime}\right)$ for $q<n$ and $H_{n}\left(M^{\prime}\right) \approx H_{n}(M) /\{u\}$, where $u$ is the homology class represented by $g\left(S^{n}, 0\right)$. If $2 n=m-1$ or $m$ the outcome is more complicated. If $m=2 n, M$ is oriented and if there is a $v \in H_{n}(M)$ such that the intersection number $u \cdot v=1$, then $H_{n}\left(M^{\prime}\right) \approx H_{n}(M) /\{u, v\}$.

Suppose $M$ has a $\xi$-structure $f$ and $u$ is in the kernel of

$$
\left(f_{M}\right)_{*}: H_{n}(M) \longrightarrow H_{n}(X)
$$

( $X$ is the base of $\xi$ ). If $X$ is simply connected, $M$ can be made simply connected by a sequence of $n=0$ and $1 \xi$-surgeries. The standard procedure for killing $u$ by $\xi$-surgery proceeds through the following steps:

(i) Represent $u$ by a map $g: S^{n} \longrightarrow M$ such that $f_{M} g: S^{n} \longrightarrow X$ is homotopic to zero. If $M$ and $X$ are simply connected and $\pi_{q}(X, M)$ is zero for $q \leq n, g$ exists. (If $X=\{p t\}, M$ is $(n-1)$-connected.)

(ii) Choose $g$ so that it is a smooth embedding. If $2 n<m$ or $M$ is simply connected and $2 n=m$, such a $g$ exists.

(iii) Extend $g$ to an embedding $g: S^{n} \times D^{m-n} \longrightarrow M$. Such an extension exists if the normal bundle of $S^{n}$ in $M, \nu$, is trivial. Since $f_{M} g$ is 
homotopic to zero, $\nu$ is stably trivial. Hence $\nu$ is trivial if $2 n<m$, or $2 n=m=4 a$ and the Euler class of $\nu$ is zero, or $2 n=m=4 a+2$ and from the two possibilities for $\nu$, trivial or $\tau\left(S^{n}\right)$, it is trivial. This last case is what this paper is all about.

(iv) Extend the $\xi$ structure over the cobordism $N$. This follows from the hypotheses in (ii), except when $m=2 n$ and $n=1,3,7$.

\section{Application of surgery to the Calculation of $b P_{m}$}

Suppose $M$ has an $0^{k}$-structure and the boundary of $M$ is null or a homotopy sphere. Starting in dimension zero, one can make it $([\mathrm{m} / 2]-1)$ connected by a sequence of $0^{k}$-surgeries. When $m=2 n+1$, delicate arguments show that $0^{k}$-surgery can be applied to produce an $n$-connected manifold and hence, by Poincaré duality, an $(m-1)$-connected manifold which is either an $m$-disc or a homotopy $m$-sphere. Hence $b P_{2 n+1}=0$ and coker $\Psi=0$ in odd dimensions.

Suppose $m=2 n$ and $M$ is $(n-1)$-connected. We first consider the case $n$ even, which provides techniques and results which one tries to mimic when $n$ is odd. As we described above, we can kill $u \in H_{n}(M ; \mathbb{Z})$ by $0^{k}$-surgery if there is a class $u \in H_{n}(M)$ such that $u \cdot v=1$ and, when $u$ is represented by an embedded $n$-sphere, its normal bundle, $\nu$, is trivial; $\nu$ is trivial if and only if its Euler class is zero if and only if $u \cdot u=0$. Thus one can kill $H_{n}(M)$ by surgery if and only if $H_{n}(M)$ has a basis $u_{i}, v_{i}, i=1,2, \ldots, r$, such that $u_{i} \cdot u_{j}=v_{i} \cdot v_{j}=0$ and $u_{i} \cdot v_{j}=\delta_{i, j}$, that is, a symplectic basis. From this one can deduce that $M$ can be made $n$-connected (and hence $m-1$ connected) if and only if the index of $M$, that is, the signature of the quadratic form on $H_{n}(M ; \mathbb{Z})$ given by the intersection pairing, is zero ([15]). For $M$ closed, the Hirzebruch index theorem expresses the index of $M$ as polynomial in the Pontrjagin classes. But since $M$ has an $0^{k}$ structure, the Pontrjagin classes are zero. Thus the cokernel of $\Psi$ is zero in dimensions $4 a, a>1$. Kervaire-Milnor also use the Hirzebruch index theorem to calculate $b P_{4 a}$.

Now suppose $M$ is as above with $n$ odd, $n \neq 1,3$ or 7 . In the $1,3,7$ cases there is an obstruction to extending the $0^{k}$-structure over the cobordism $N$. Although our function $\phi$ measures this obstruction, we do not treat this case because of the difficulty of the surgery details required. Suppose $M$ is $(n-1)$-connected. Let $\phi: H_{n}(M ; \mathbb{Z}) \longrightarrow \mathbb{Z}_{2}=\mathbb{Z} / 2 \mathbb{Z}$ be defined as follows: For $u \in H_{n}(M ; \mathbb{Z})$, represent $u$ by an embedded $n$-sphere and let $\nu_{u}$ be its normal bundle in $M$. Let $\phi(u)=0$ or 1 according as $\nu_{u}$ is trivial or isomorphic to $\tau\left(S^{n}\right)$ (the only two possibilities for $\nu_{u}$ ).

Lemma 5.1. $\phi$ is well defined and satisfies:

$$
\phi(u+v)=\phi(u)+\phi(v)+u \cdot v .
$$


Since $n$ is odd, $u \cdot u=0$, and therefore $\phi(2 u)=0$. Hence we do not lose anything by taking $u$ in $H_{n}\left(M ; \mathbb{Z}_{2}\right)$. For the remainder of this paper $H_{n}(M)=H_{n}\left(M ; \mathbb{Z}_{2}\right)$. As above, we may make $M(m-1)$-connected by $0^{k}$-surgery if $H_{n}(M)$ has a symplectic basis $u_{i}, v_{i}$ such that $\phi\left(u_{i}\right)=0$ for all $i$. Arf associated to quadratic functions such as $\phi$ a $\mathbb{Z}_{2}$ invariant given by

$$
A(\phi)=\sum \phi\left(u_{i}\right) \phi\left(v_{i}\right)
$$

He also proved that given the pairing, $A(\phi)$ classifies such quadratic functions and $H_{n}(M)$ has a symplectic basis $u_{i}, v_{i}$ such that $\phi\left(u_{i}\right)=0$, for all $i$, if and only if $A(\phi)=0$. Thus, if $A(\phi)=0, M$ can be made $(2 n-1)$ connected. Starting with a closed $2 n$-manifold, $n$ odd $\neq 1,3,7$ and $0^{k}$ structure $f$ on $M$, by a sequence of surgeries one can produce an $(n-1)$ connected $\left(M^{\prime}, f^{\prime}\right)$ and then a $\phi_{M^{\prime}}$. Then $A\left(\phi_{M^{\prime}}\right)$ is the Kervaire invariant of $M$ and the following was proved in [10]:

Theorem 5.2. Sending $(M, f)$ to $A\left(\phi_{M^{\prime}}\right)$ induces a homomorphism

$$
\alpha: \Omega_{2 n}\left(0^{k}\right) \longrightarrow \mathbb{Z}_{2}
$$

An element $z \in \Omega_{2 n}\left(0^{k}\right)$ can be represented by a homotopy sphere, if and only if $\alpha(z)=0$.

Corollary 5.3. In dimensions $4 a+2$, the kernel and cokernel of $\Psi$ are 0 or $\mathbb{Z}_{2} ;$ ker $\Psi=0$ if and only if $\alpha=0$ and coker $\Psi=0$ if and only if $\alpha \neq 0$.

The present state of knowledge on $\alpha$ is:

Theorem 5.4. $\alpha \neq 0$ for $2 n=2,6,14$ ([11]), 30 ([24]), $62([2])$ and $\alpha=0$ for $2 n=10([10]), 8 a+2([7]), \neq 2^{j}-2([3]) . \quad \alpha \neq 0$ if and only if $h_{j}^{2}$ lives to $E_{\infty}$ in the Adams spectral sequence for stable homotopy groups of spheres. ([3]) (Should such an element exist it is called $\theta_{j}$.)

We prove Browder's results in section 8.

An $(n-1)$-connected $2 n$-manifold $M$ with $0^{k}$-structure, boundary a homotopy sphere and $A\left(\phi_{M}\right)=1$ may be constructed as follows: One plumbs together two copies of $D \tau\left(S^{n}\right)$ as follows: Let $h: D^{n} \longrightarrow S^{n}$ be a homeomorphism onto the upper hemisphere of $S^{n}$ given by $h(x)=\left(x, \sqrt{1-|x|^{2}}\right)$ and $r: D^{n} \times D^{n} \longrightarrow \tau\left(S^{n}\right)$ be a bundle map covering $h$. Let $M$ be a smoothed version of $D \tau\left(S^{n}\right) \times\{0\} \cup D \tau\left(S^{n}\right) \times\{1\}$ with $(h(x, y), 0)$ identified with $(h(y, x), 1)$ for all $x, y$. An easy cell decomposition of $M$ shows that its boundary is two $n$-discs glued together along their boundaries and hence the boundary of $M$ is a homotopy sphere $\Sigma$. Let $N$ be $M$ with $\Sigma$ coned off. 
Theorem 5.5. If $\Sigma$ is diffeomorphic to $S^{2 n-1}, N$ is smoothable and has Kervaire invariant one; otherwise $N$ is a topological manifold which does not admit a differentiable structure $([10])$ and $\Sigma$ generates $b P_{2 n} \approx \mathbb{Z}_{2}$.

The proofs of the results cited in theorem 5.4 follow a very orderly path which we now outline. We switch from homology to cohomology, $H^{n}(M)=H^{n}\left(M ; \mathbb{Z}_{2}\right)$. Composing with Poincaré duality, $\phi$ becomes $\phi$ : $H^{n}(M) \longrightarrow \mathbb{Z}_{2}$. One wants to associate to a closed $2 n$-manifold $M, n$ odd, $\phi$ satisfying:

(5.6) $\phi(u+v)=\phi(u)+\phi(v)+u v([M])$ where $u v$ denotes cup product.

(5.7) If the Poincaré dual of $u$ can be represented by an embedded $n$ sphere with stably trivial normal bundle $\nu$ and $n \neq 1,3,7$, then $\phi(u)=0$ if and only if $\nu$ is trivial.

In [10] Kervaire defines $\phi$ for an $(n-1)$-connected $M$ as follows. Recall the loop space $\Omega=\Omega\left(S^{n+1}\right)$ has cohomology generators $e_{i}$ in dimensions $n i$ and under the multiplication $\Omega \times \Omega \longrightarrow \Omega, e_{1}$ goes to $e_{1} \otimes 1+1 \otimes e_{1}$ and $e_{2}$ goes to $e_{2} \otimes 1+1 \otimes e_{2}+e_{1} \otimes e_{1}$. For $u \in H^{n}(M)$ there is a map $f_{u}: M \longrightarrow \Omega$ such that $f_{u}^{*}\left(e_{1}\right)=u$; $\phi$ is then defined by $\phi(u)=f_{u}^{*}\left(e_{2}\right)$ and satisfies 5.6 and 5.7. Then it is shown that $\alpha: \pi_{2 n+k}\left(S^{k}\right)=\Omega_{2 n} \longrightarrow \mathbb{Z}_{2}$, as above, is a well defined homomorphism. Kervaire proves that $\alpha$ is zero for $n=5$ using knowledge of $\pi_{10+k}\left(S^{k}\right)$, namely, this group has a unique element $a$ of order two and $a=b c, b \in \pi_{k+1}\left(S^{k}\right)$ which represents a manifold $S^{1} \times M^{\prime}$ which then can be surgered to a homotopy sphere. We remark that an equivalent way of defining $\phi$ would be to represent $u$ by a map $F: S M \longrightarrow S_{n+1}$ ( $S=$ suspension) and define $\phi(u)$ to be the functional squaring operation $S q_{F}^{n+1}\left(s_{n+1}\right), s_{n+1}$ the cohomology generator.

In [5] $\alpha: \Omega_{8 a+2}($ Spin $) \longrightarrow \mathbb{Z}_{2}$ was defined as follows: $\Omega_{m}($ Spin $)=\Omega_{m}(\xi)$, where $\xi \longrightarrow B \operatorname{Spin}_{k}$ is the universal $\operatorname{Spin}_{k}$ vector bundle. For $n=4 a+1$, the Adem relation,

$$
S q^{n+1}=S q^{2} S q^{n-1}+S q^{1} S q^{2} S q^{n-2}
$$

gives a relation on $H^{n}(M)$,

$$
S q^{2} S q^{n-1}+S q^{1} S q^{2} S q^{n-2}=0
$$

which in turn gives a secondary cohomology operation on $M$ with a Spin structure,

$$
\phi^{\prime}: H^{n}(M) \longrightarrow H^{2 n}(M) .
$$

Define $\phi(u)=\phi^{\prime}(u)(M)$. The Spin structure is used to ensure that $\phi^{\prime}$ is defined on all of $H^{n}(M)$ with zero indeterminacy. For example, $S q^{1}$ : $H^{2 n-1}(M) \longrightarrow H^{2 n}(M)$ is given by $S q^{1}(v)=w_{1} v$. Then $\phi$ satisfies 5.6 
and 5.7 and defines $\alpha$. In [7] we showed that $\alpha$ was zero on the image of $\Omega_{8 k+2}\left(0^{k}\right)$ (framed cobordism) in $\Omega_{8 a+2}$ (Spin) using the result of Conner and Floyd and Lashof and Rothenberg that if $A \in \Omega_{8 k+2}(S U)$ goes to zero in $\Omega_{8 k+2}(U)$, then $A=B^{2} C, B \in \Omega_{8 k}(S U)$.

In [3] Browder developed $\alpha: \Omega_{2 n}(\xi) \longrightarrow \mathbb{Z}_{2}, n$ even or odd, $\xi \longrightarrow X$, as follows: One may assume that $X$ is a smooth closed $N$-manifold, $N$ large, with normal bundle in $\mathbb{R}^{N+k}$ equal to $\xi$. Suppose $M$ is a smooth closed $2 n$-manifold with $\xi$-structure $f$. One may assume $f_{M}$ is an embedding. Then the normal bundle of $M$ in $X$ is trivial and trivializing and using the Thom construction one obtains a map $F: X \longrightarrow S^{k} M\left(S^{k} M\right.$, the $k$-fold suspension of $M)$. Then $\phi$ is defined by

$$
\phi(u)=S q_{F}^{n+1}\left(S^{k} u\right) .
$$

In order for $\phi(u)$ to be defined, $F^{*}\left(S^{k} u\right)$ must equal zero, and for there to be no indeterminacy, $S q^{n+1}: H^{N-n-1}(X) \longrightarrow H^{N}(X)$ must be zero. $S q^{n+1}(w)=v_{n+1} w$ for $w \in H^{N-n-1}(X)$, where $v_{n+1}$ is the Wu class of $\xi$. One restricts the choice of $\xi$ to bundles with $v_{n+1}=0$. Then $\phi: P \longrightarrow \mathbb{Z}_{2}$ where $P=\left\{u \in H^{n}(M) \mid F^{*}\left(S^{k} u\right)=0\right\}$. Where defined $\phi$ satisfies 5.6 and 5.7. One restricts $\alpha$ to those $(M, f)$ such that $\phi$ is zero on all $u \in P$ such that $u v(M)=0$ for all $v \in P$. The Arf invariant algebra then works to give an integer mod 2. Then $\alpha$ is related to the Adams spectral sequence by computing a Postnikov system, up to the relevant dimension of $M O\left[v_{n+1}\right]$. We give the details in section 8 .

\section{Generalized Groups of Homotopy Spheres}

Several people, most notably Novikov, discovered that the Groups of Homotopy Spheres paper ([11]) could be generalized by the following two step process. Replace $0^{k}$, the bundle $\mathbb{R}^{k} \longrightarrow p t$, by $O_{m}^{k}$ the bundle $S^{m} \times$ $\mathbb{R}^{k} \longrightarrow S^{m}$. Then the coker $\Psi$ question asks: "Which elements $\{M, f\}$, where $f_{M}$ has degree one, can be represented by $\{M, f\}$, where $f_{M}$ is a homotopy equivalence?" (For there to be an $(M, f)$ with $f_{M}$ of degree one, the top homology class of $T(\xi)$ must be spherical.) The $b P_{m+1}$ question asks, "If $\{M, f\}=\left\{S^{m}, i d\right\}$, can the cobordism between them be chosen to be an $h$-cobordism?" Now replace all occurrences of $O_{m}^{k}$ with $\xi \longrightarrow$ $X$, where $X$ is a simply connected CW complex of finite type satisfying Poincaré duality in dimension $m$, that is there a class $x \in H_{m}(X ; \mathbb{Z})$ such that cap product with $x$ gives an isomorphism, $H^{n}(X) \longrightarrow H_{m-n}(X)$ for all $n$. Then everything in Kervaire-Milnor goes through. Suppose $\{M, f\} \in$ $\Omega_{m}(\xi)$ and $f_{M}$ has degree one. The trick is to consider the commutative diagram: 


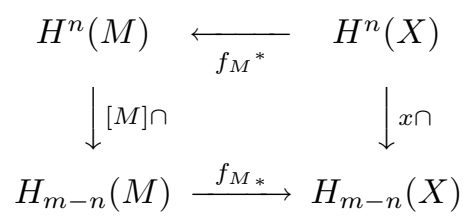

By Poincaré duality $f_{M_{*}}$ is an epimorphism and $f_{M}{ }^{*}$ is a monomorphism. For $2 n<m$ one can kill elements in the kernel of $f_{M_{*}}$ just as in [11]. This material is thoroughly described in Browder's book "Surgery on Simply Connected Manifolds" ([4]). This material, when $X$ is not simply connected, is the subject of Wall's book "Surgery on Compact Manifolds" $([22])$, where the general pattern of the above is followed but surgery in the middle dimensions is much more complicated and leads to Wall's $L$ groups, in which the obstructions to doing the middle dimension surgery lie. Ranicki develops in "Exact Sequences in the Algebraic Theory of Surgery" ([19]) a completely algebraic approach to these surgery obstructions, replacing manifolds by their chain complex analogs. Michael Weiss refines this algebraic approach to surgery obstructions and makes it more calculable $([23],[24])$.

\section{A Further Generalization of $\phi$}

We state the main theorems of this section and then prove them.

In the remainder of this section most spaces have base points, $M^{+}$is $M$ with a disjoint base point, $[X, Y]$ is the homotopy classes of maps from $X$ to $Y,\{X, Y\}=\lim \left[S^{k} X, S^{k} Y\right]$ and $\eta:[X, Y] \longrightarrow\{X, Y\}$ sends $[f]$ to $\{f\}$. Let $s: S^{n} \longrightarrow K_{n}$ be the $\pi_{*}$ generator.

Lemma 7.1. The Hopf construction $h(\lambda): S^{2 n+1} \longrightarrow S K_{n}$ on

$$
\lambda: S^{n} \times S^{n} \stackrel{s \times s}{\longrightarrow} K_{n} \times K_{n} \stackrel{\mu}{\longrightarrow} K_{n}
$$

with $\mu$ the multiplication, gives a generator of $\left\{S^{2 n}, K_{n}\right\} \approx \mathbb{Z}_{2}$.

Suppose $M$ is a closed $2 n$-manifold ( $n$ even or odd). We form an abelian group $G(M)=H^{n}(M) \times H^{2 n}(M)$ with addition

$$
(u, v)+\left(u^{\prime}, v^{\prime}\right)=\left(u+u^{\prime}, v+v^{\prime}+u u^{\prime}\right) .
$$

Let $j: \mathbb{Z}_{2} \longrightarrow \mathbb{Z}_{4}$ be the homomorphism sending 1 to 2 . Then functions $\phi: H^{n}(M) \longrightarrow \mathbb{Z}_{4}$ satisfying $\phi(u+v)=\phi(u)+\phi(v)+j(u v([M]))$ are in one to one correspondence with homomorphisms $h: G(M) \longrightarrow \mathbb{Z}_{4}$ such that $h(0, v)=j(v([M]))$. We will see that such functions occur in nature. 
Theorem 7.2. Let $F: G(M) \longrightarrow\left\{M^{+}, K_{n}\right\}$, given by $F(u, v)=\eta(u)+$ $h(\lambda) g_{v}$, where $g_{v} \longrightarrow S^{2 n}$ has degree one. Then $F$ is an isomorphism.

Let $\nu$ be the normal bundle of $M$ in $\mathbb{R}^{2 n+k}$ and $\Delta: T \nu \longrightarrow T \nu \wedge M^{+}$ be the diagonal map sending $v$ to $(v, p(v)), p: \nu \longrightarrow M$. Then $S^{2 n+k} \stackrel{t}{\rightarrow}$ $T \nu \stackrel{\Delta}{\longrightarrow} T \nu \wedge M^{+}$is an $S$ (Spanier-Whitehead) duality map ([20]). Then $\left\{M^{+}, K_{n}\right\} \approx\left\{S^{2} n, T \nu \wedge K_{n}\right\}$ under the map sending $S^{l} M \longrightarrow S^{l} K_{n}$ to

$$
S^{2 n+k+l} \longrightarrow S^{l} T \nu \longrightarrow S^{l}\left(T \nu \wedge M^{+}\right)=T \nu \wedge S^{l} M^{+} \longrightarrow T \nu \wedge S^{l} K_{n}
$$

Let $q(\lambda) \in\left\{S^{2 n}, T \nu \wedge K_{n}\right\}$ be the image of $h(\lambda)$ under this map.

Lemma 7.3. If $f: \nu \longrightarrow \xi$, the image of $f_{*}(q(\lambda))$ is non-zero if and only if $v_{n+1}(\xi)=0$ and it is at most divisible by 2 .

We call $\xi$ a Wu- $n$ spectrum if $v_{n+1}(\xi)=0$ in which case we can choose a homomorphism $\omega:\left\{S^{2 n}, T \xi \wedge K_{n}\right\} \longrightarrow \mathbb{Z}_{4}$ such that $\omega\left(\left(f_{*}(q(\lambda))\right)=2\right.$. Let $\phi=\phi(M, f, \omega)$ be the composition $H^{n}(M)=\left[M^{+}, K_{n}\right] \longrightarrow\left\{M^{+}, K_{n}\right\} \longrightarrow$ $\left\{S^{2 n}, T \nu \wedge K_{n}\right\} \longrightarrow\left\{S^{2 n}, T \xi \wedge K_{n}\right\} \longrightarrow \mathbb{Z}_{4}$. Hence,

Theorem 7.4. $\phi=\phi(M, f, \omega)$ satisfies

$$
\phi(u+v)=\phi(u)+\phi(v)+j(u v([M])) .
$$

Let $B O_{k}\left[v_{n+1}\right] \longrightarrow B O_{k}$ be the fibration with fibre $K_{n}$ and $k$-invariant $v_{n+1}$ and let $\xi\left[v_{n+1}\right]$ be the pull back of the universal bundle over $B O_{k}$. Suppose $M$ has a $\xi\left[v_{n+1}\right]$-structure, $S^{n} \subset M$ has normal bundle $\mu$ and $\nu \mid S^{n}$ is trivial. Then $S^{n} \longrightarrow M \longrightarrow B O_{k}\left[v_{n+1}\right]$ factors through $K_{n}$. Let $\epsilon_{1}=0$ or $1=$ degree of this map. Let $\epsilon_{2}=0$ or 1 according to whether $\mu$ is trivial or $\tau\left(S^{n}\right)$. Let $u$ be the Poincaré dual of the homology class represented by $S^{n} \longrightarrow M$.

Lemma 7.5. If $n$ is odd and $\neq 1,3,7$, then $\phi(u)=2\left(\epsilon_{1}+\epsilon_{2}\right)$.

Remark. If $n$ is even, $\epsilon_{2}=$ Euler number of $\mu \bmod 4$. If $n=1,3$, or 7 and $\epsilon_{2}=0$, the element in $\pi_{2 n+k}\left(T \xi \wedge K_{n}\right)$ involves a map $g: S^{2 n+k} \longrightarrow S^{k}$ such that $\epsilon_{1}=$ Hopf invariant of $g$.

Let $S^{n} \times S^{n} \longrightarrow B O_{k}\left[v_{n+1}\right]$ be the composition of $K_{n} \longrightarrow B O_{k}\left[v_{n+1}\right]$ and $s \otimes 1+1 \otimes s$. This lifts to a $\xi\left[v_{n+1}\right]$-structure $q$. Then 7.5 gives

Lemma 7.6. The $\phi$ associated to $\left(S^{n} \times S^{n}, q\right)$. satisfies $\phi(s \otimes 1)=\phi(1 \otimes$ $s)=2$.

The following gives an analog of the Arf invariant for these quadratic functions. 
Definition 7.7. Let $V$ be a finite dimensional vector space over $\mathbb{Z}_{2}$. A function $\phi: V \longrightarrow \mathbb{Z}_{4}$ is a (nonsingular) quadratic if it satisfies $\phi(u+$ $v)=\phi(u)+\phi(v)+j t(u, v)$ where $j: \mathbb{Z}_{2} \longrightarrow \mathbb{Z}_{4}$ sends 1 to 2 and $t$ is a nonsingular bilinear pairing. If $\phi_{i}: V_{i} \longrightarrow \mathbb{Z}_{4}, i=1,2$, are such functions $\phi_{1} \approx \phi_{2}$ if there is an isomorphism $T: V_{1} \approx V_{2}$ such that $\phi_{1}=\phi_{2} T$. $\left(\phi_{1}+\phi_{2}\right): V_{1}+V_{2} \longrightarrow \mathbb{Z}_{4},-\phi$ and $\left(\phi_{1} \phi_{2}\right): V_{1} \otimes V_{2} \longrightarrow \mathbb{Z}_{4}$ are defined by $\left(\phi_{1}+\phi_{2}\right)(u, v)=\phi_{1}(u)+\phi_{2}(v),(-\phi)(u)=-\phi(u)$ and $\left(\phi_{1} \phi_{2}\right)(u \otimes v)=$ $\phi_{1}(u) \phi_{2}(v)$.

A proof of the following appears in [6] and is straightforward. The first part of the theorem is proved by showing that the Grothendieck group of these functions is cyclic of order eight.

Theorem 7.8. There is a unique function $\sigma$ from quadratic functions as in 7.1 to $\mathbb{Z}_{8}$ satisfying:

(i) If $\phi_{1} \approx \phi_{2}$, then $\sigma\left(\phi_{1}\right)=\sigma\left(\phi_{2}\right)$

(ii) $\sigma\left(\phi_{1}+\phi_{2}\right)=\sigma\left(\phi_{1}\right)+\sigma\left(\phi_{2}\right)$

(iii) $\sigma(-\phi)=-\sigma(\phi)$

(iv) $\sigma(\gamma)=1$, where $\gamma: \mathbb{Z}_{2} \longrightarrow \mathbb{Z}_{4}$ by $\gamma(0)=0$ and $\gamma(1)=1$.

Furthermore $\sigma$ satisfies:

(v) If $\phi=j \phi^{\prime}, \sigma(\phi)=4 \operatorname{Arf}\left(\phi^{\prime}\right)$.

(vi) If $\phi: V \longrightarrow \mathbb{Z}_{4}, \sigma(\phi)=\operatorname{dim} V \bmod 2$.

(vii) $\sigma\left(\phi_{1} \phi_{2}\right)=\sigma\left(\phi_{1}\right) \sigma\left(\phi_{2}\right)$.

(viii) If $U$ is a finitely generated abelian group, $\tau: U \otimes U \longrightarrow \mathbb{Z}$ is a symmetric unimodular form, $\Psi(u)=\tau(u, u)$ and $\phi: U / 2 U \longrightarrow \mathbb{Z}_{4}$ is defined by $\phi(u)=\Psi(u) \bmod 4$, then $\phi$ is quadratic and $\sigma(\phi)=$ (signature $\Psi$ ) $\bmod 8$.

(ix) Suppose $t$ is the bilinear form of $\phi: V \longrightarrow \mathbb{Z}_{4}, V_{1} \stackrel{\nu}{\rightarrow} \nu V \stackrel{\delta}{\rightarrow} V_{2}$ is an exact sequence and $t^{\prime}: V_{1} \otimes V_{2} \longrightarrow \mathbb{Z}_{2}$ is a nonsingular bilinear form such that $t^{\prime}(u, \delta v)=t(\nu u, v)$. If $\phi \nu=0$, then $\sigma(\phi)=0$.

(x) With $i=\sqrt{-1}$,

$$
\sigma(\phi)=(4 i / \pi) \ln \left(2^{(\operatorname{dim} V) / 2} /\left(\sum_{u \in V} i^{\phi(u)}\right)\right) .
$$

Theorem 7.9. Sending $(M, f)$ to $\sigma(\phi(M, f, \omega))$ induces a homomorphism $\sigma_{\omega}: \Omega_{2 n}\left(\xi\left[v_{n+1}\right]\right) \longrightarrow \mathbb{Z}_{8}$ such that $\sigma_{\omega}$ composed with

$$
\Omega_{2 n}\left(0^{k}\right) \longrightarrow \Omega_{2 n}\left(\xi\left[v_{n+1}\right]\right)
$$

gives the Kervaire invariant.

We can apply $\sigma_{\omega}$ to the general, simply connected surgery problem as follows. 
Definition 7.10. For $n$ odd, a $2 n$-Poincaré quadruple $(X, \xi, \beta, \omega)$ is a connected finite $C W$ complex $X$, a k-plane bundle $\xi$ over $X, \omega$ a homomorphism as above and $\beta \in \pi_{m+k}(T \xi)$ such that

$$
S^{m+k} \stackrel{\beta}{\longrightarrow} T \xi \stackrel{\Delta}{\longrightarrow} T \xi \wedge X_{+}
$$

is an $S$ duality (which makes $\xi$ a Wu bundle).

Theorem 7.10. Then by the Thom Theorem, $\beta$ gives a $2 n$-manifold $M$ with a $\xi$-structure $f$ such that $f_{M}$ has degree one and the surgery obstruction to making $f_{M}$ a homotopy equivalence is $\sigma\left(\phi\left(X, i d_{X}, \omega\right)\right)-\sigma(\phi(M, f, \omega))$.

Proof of 7.1 and 7.2. Let $\iota$ be the generator of $H^{n}\left(K_{n}\right)$. Let $E \longrightarrow K_{n+1}$ be the fibration with fibre $K_{2 n+1}$ and $k$-invariant $\iota_{n+1}^{2}$. Then $S \iota_{n} \longrightarrow K_{n+1}$ lifts $g: S \iota_{n} \longrightarrow E$ and on homotopy groups $\pi_{i} \pi_{i}(g)$ is an isomorphism for $i \leq 2 n+1$. Then since $M$ is $2 n$ dimensional $\left\{M, K_{n}\right\}=\left[S M, S K_{n}\right] \approx$ $[S M, E] \approx[M, \Omega E]=\left[M, K_{n} \times K_{2 n}\right]=H^{n}(M) \times H^{2 n}(M)$. The additive structure comes from the fact that under multiplication $\Omega E \times \Omega E \longrightarrow$ $\Omega E, \iota_{2 n}$ goes to $\iota_{2 n} \otimes 1+1 \otimes \iota_{2 n}+\iota_{n} \otimes \iota_{n}$. Applying this to $M=S^{n} \times S^{n}$ gives 7.1 .

Proof of 7.3. Let $V(X)=\left\{S^{2 n+k+l}, X \wedge S^{l}\left(K_{n}\right)\right\}$. We want to know the image of $V\left(S^{k}\right)$ in $V(T \xi)$ and how divisible it is. We can assume $T \xi$ is a finite $(k-1)$-connected CW complex and $S^{l}\left(K_{n}\right) \longrightarrow K_{n+l}$ is a fibration with fiber $K_{2 n+l}$ and $k$-invariant $S q^{n+1} \iota_{n+l}$. Applying $\left\{S^{2 n+k+l}, T \xi \wedge()\right\}$, this gives an exact sequence

$$
\longrightarrow H_{k+n+1}(T \xi) \stackrel{S q^{n+1}}{\longrightarrow} H_{k}(T \xi) \longrightarrow V(T \xi) \longrightarrow H_{k+n}(T \xi) \longrightarrow
$$

and the same with $T \xi$ replaced by $S^{k}$. For $x \in H_{k+n+1}(T \xi)$ and $U$ the Thom class,

$$
U\left(S q^{n+1}(x)\right)=\chi\left(S q^{n+1}\right)(U)(x)=v_{n+1} U(x) .
$$

The two exact sequences make a ladder from which the desired result can be read off.

Proof of 7.5. Note, applying the Thom construction and the Thom class give maps $S^{n} \times S^{n} \longrightarrow T \tau\left(S^{n}\right) \longrightarrow K_{n}$ and the Hopf construction gives $S^{2 n+1} \longrightarrow S T \tau\left(S^{n}\right) \longrightarrow S K_{n}$. Also the element $a \in \pi_{2 n+k}\left(T \xi \wedge K_{n}\right)$ such that $\phi(u)$ comes from it is given by $S^{2 n+k} \longrightarrow T \nu_{S^{n}} \longrightarrow S^{k}\left(S^{n+}\right) \wedge T \mu \longrightarrow$ $S^{k}\left(K_{n}\right) \wedge K_{n}=S^{k} K_{n} \vee S^{k} K_{n} \wedge K_{n} \longrightarrow T \xi \wedge K_{n}$. Combining these two gives the desired result. 
Proof of 7.9. To show that $\sigma(\omega)$ is well defined suppose $(M, f)=\partial(N, F)$. By virtue of 7.8(ix) and the exact sequence

$$
H^{n}(N) \stackrel{j^{*}}{\longrightarrow} H^{n}(M) \longrightarrow H^{n+1}(N, M),
$$

it is sufficient to show that $\phi\left(j^{*}(u)=0\right.$. The element $a \in \pi_{2 n+k}\left(T \xi \wedge K_{n}\right)$ such that $\phi\left(j^{*}(u)=\omega(a)\right.$ is

$$
\begin{aligned}
S^{2 n+k+1} \longrightarrow & T \nu_{N} / T \nu_{M} \longrightarrow T \nu_{N} \wedge(N / M) \longrightarrow \\
& T \nu_{N} \wedge S M^{+} \longrightarrow T \nu_{N} \wedge S N^{+} \longrightarrow T \xi \wedge S K_{n}
\end{aligned}
$$

But this is zero because $N / M \longrightarrow S M^{+} \longrightarrow S N^{+}$is zero.

\section{Proof of Theorem 5.4}

Let $B=B O_{k}, K_{n}=K\left(\mathbb{Z}_{2}, n\right), v_{n+1}: B \longrightarrow K_{n+1}$ represent the $(n+1)$ th Wu class, $C=\left\{(b, a) \in B \times K_{n+1}^{I} \mid a(1)=v_{n+1}(b)\right\}, D=B O_{k}\left[v_{n+1}\right]=$ $\{(b, a) \in C \mid a(0)=*\}, i: B \longrightarrow C$ by $i(b)=\left(b, a_{b}\right)$ where $a_{b}(t)=v_{n+1}(b)$ and $\pi:(C, D) \longrightarrow\left(B \times K_{n+1}, B \times\{*\}\right)$ and $\pi^{\prime}: C \longrightarrow B \times K_{n+1}$ by $(b, a) \rightarrow(b, a(0))$. The following is easily verified:

Lemma 8.1. The map $i$ is a homotopy equivalence. $\pi$ is a fibre map with fibre $K_{n}$ and in the Serre cohomology spectral sequence for $\pi, E_{2}^{p, q}=0$ for $q>0$ and $p+q \leq 2 n+2$ except $E_{2}^{n+1, n} \approx \mathbb{Z}_{2}$. Hence $\pi^{*}: H^{p}\left(B \times K_{n+1}, B \times\right.$ $\{*\}) \approx H^{p}(C, D)$ for $p \leq 2 n+2$ except it may have a kernel isomorphic to $\mathbb{Z}_{2}$ for $p=2 n+2$.

Lemma 8.2. The kernel of

$$
\pi^{*}: H^{2 n+2}\left(B \times K_{n+1}, B \times\{*\}\right) \longrightarrow H^{2 n+2}(C, D)
$$

is generated by $v_{n+1} \otimes \iota_{n+1}+1 \otimes \iota_{n+1}^{2}$.

Proof. Recall that via the inclusion map $j: X \longrightarrow(X, A), H^{*}(X)$ acts on $H^{*}(X, A)$, and for $u \in H^{*}(X, A), u^{2}=j^{*}(u) u$. Note $i \pi^{\prime}$ sends $b$ to $\left(b, v_{n+1}(b)\right)$ and hence $\left(i \pi^{\prime}\right)^{*}\left(1 \otimes \iota_{n+1}\right)=v_{n+1}$. Thinking of $1 \otimes \iota_{n+1} \in$ $H^{n+1}\left(B \times K_{n+1}, B \times\{*\}\right)$,

$$
\begin{aligned}
\pi^{*}\left(1 \otimes \iota_{n+1}^{2}\right) & =\pi^{\prime *} j^{*}\left(1 \otimes \iota_{n+1}\right)\left(1 \otimes \iota_{n+1}\right) \\
& =\pi^{*}\left(v_{n+1} \otimes 1\right)\left(1 \otimes \iota_{n+1}\right) \\
& =\pi^{*}\left(v_{n+1} \otimes \iota_{n+1}\right) .
\end{aligned}
$$

Under the map $C \longrightarrow B$ sending $(b, a)$ to $b$, the universal bundle over $B$ pulls back to bundles over $C$ and $D$ whose Thom spaces we denote by $T D, T C$, and $T B$ and whose Thom classes we denote by $U$. The map $\pi$ induces a map $T \pi: T C \wedge T D \longrightarrow T B \wedge K_{n+1}$. And, 
Lemma 8.3. The kernel of $H^{q}(T \pi)$ for $q \leq 2 n+2+k$ is generated by

$$
\begin{aligned}
v_{n+1} U \otimes \iota_{n+1}+U \otimes \iota_{n+1}^{2}=\chi\left(S q^{n+1}\right) & U \otimes \iota_{n+1}+1 \otimes \iota_{n+1}^{2} \\
& =\sum_{i>0} \chi\left(S q^{i}\right)\left(U \otimes S q^{n+1-i} \iota_{n+1}\right) .
\end{aligned}
$$

The same Steenrod square manipulations yield:

Lemma 8.4. For $j<n+1, T B \longrightarrow T C \longrightarrow T C / T B \longrightarrow T C \wedge K_{n+1}$ sends $\left(U \otimes S q^{j} \iota_{n+1}\right)$ to

$$
\sum_{k>0}\left(S q^{k}\left(v_{n+1} v_{j-k} U\right)+\chi\left(S q^{k}\right)\left(\left(S q^{j+1-k} v_{j}\right) U\right)\right) .
$$

We very briefly describe the portion of Adams' work on cohomology operations ([1]) relevant to this proof. All the spaces we will deal with will be approximately $k$-connected, and the results we state will be correct in a range of dimensions up to about $2 k$. Suppose $L$ and $K$ are spaces and $F: L \longrightarrow K$ is a map, $E$ is the space of paths in $L$ starting at a base point and $P: E \longrightarrow L$ sends a path to its end point. Note $E$ is contractible and $P$ is a fibre map with fibre the loops on $K, \Omega(K)$. Let $p: E_{F}=F^{*} E \longrightarrow L$ be the induced fibration. Suppose $G_{1}: L_{1} \longleftarrow L_{2}$ is an inclusion and $F: L_{2} \longrightarrow L_{2} / L_{1}$ is the quotient map. Then $L_{1}=E_{F}$ and $p=G$. Suppose $G_{2}: L_{2} \longrightarrow L_{3}$ and $G_{2} G_{1}$ is homotopic to the constant map. Then $G_{1}$ lifts to $G_{1}^{\prime}: L_{1} \longrightarrow E_{G_{2}}$ and we form $E_{G_{1}^{\prime}}$. There is a map $G_{2}^{\prime}: E_{G_{1}} \longrightarrow \Omega\left(L_{2}\right)$ such that $E_{G_{2}^{\prime}}=E_{G_{1}^{\prime}}$. Call this space $E\left(G_{1}, G_{2}\right)$. We apply this to our situation by taking $L_{1}=T B$, $L_{2}=T B \wedge K_{n+1}, L_{3}=K_{2 n+2+k}, G_{1}: T B \longrightarrow T C / T D \longrightarrow T B \wedge K_{n+1}$ and $G_{2}: T B \wedge K_{n+1} \longrightarrow K_{2 n+2+k}$ representing the cohomology element in Lemma 8.3. The map $T D=T\left(B O_{k}\left[v_{n+1}\right]\right) \longrightarrow T\left(B O_{k}\right)=T B$ lifts to $h: T D \longrightarrow E\left(G_{1}, G_{2}\right)$, and

Lemma 8.5. $\pi_{q}(h)$ is an isomorphism for $q \leq 2 n+k$.

Proof. $T C / T D \longrightarrow T B \wedge K_{n+1}$ lifts to $r: T C / T D \longrightarrow E_{G_{2}} . H^{q}(r)$ is an isomorphism for $q \leq 2 n+2+k$, hence $\pi_{q}(r)$ is an isomorphism for $q \leq 2 n+1+k$ and hence $\pi_{q}(h)$ is as an isomorphism for $q \leq 2 n+k$.

The cohomology of both $T C=T B$ and $T B \wedge K_{n+1}$ are free modules over the mod two Steenrod algebra $A$. If $\left\{u_{i}\right\}$ is a basis for $H^{*}(T B)$ over $A$ and $\left\{v_{i}\right\}$ is a basis for $H^{*}\left(K_{n+1}\right),\left\{u_{i} \otimes v_{j}\right\}$ is a free $A$-basis for $H^{*}\left(T B \wedge K_{n+1}\right)$. Up to homotopy type $T B=\prod K_{\left|u_{i}\right|}$ and similarly for $T B \wedge K_{n+1}$.

Let $s: S^{k} \longrightarrow T D$ represent the generator of $\pi_{k}(T D)$. Then the map of $2 n$-framed cobordism to $2 n_{B} O_{k}\left[v_{n+1}\right]$ cobordism corresponds to $s_{*}$ : 
$\pi_{2 n+k}\left(S^{k}\right) \longrightarrow \pi_{2 n+k}(T D) \approx \pi_{2 n+k}\left(E\left(G_{1}, G_{2}\right)\right)$. Let $V: K_{k} \longrightarrow T B$ be the map such that $U$, the Thom class pulls back to $\iota_{k}$. Then $S^{k} \longrightarrow T D \longrightarrow$ $T B$ factors through $V: K_{k} \longrightarrow T B$ and hence $S^{k} \longrightarrow T D \longrightarrow E\left(G_{1}, G_{2}\right)$ factors through $E\left(G_{1} V, G_{2}\right)$ giving a map $t: S^{k} \longrightarrow E\left(G_{1} V, G_{2}\right)$. In [1] Adams (with some refinements) that one may of viewing $E\left(G_{1} V, G_{2}\right)$ is as the beginning of a tower building $S^{k}$. This tower gives a spectral sequence with $E_{2}=\operatorname{Ext}_{A}\left(\mathbb{Z}_{2}, \mathbb{Z}_{2}\right)$ and a map $S^{2 n+k} \longrightarrow E\left(G_{1} V, G_{2}\right)$ representing an element of $E_{2}$ is said to live to $E_{\infty}$ if it lifts all the way up the tower giving a map $S^{2 n+k} \longrightarrow S^{k}$ (more or less). At this two stage level, the relevant elements of $E_{2}$ have names " $h_{i} h_{j}$ ", $i \leq j$. Adams proves that if a map $S^{2 n+k} \longrightarrow S^{k} \longrightarrow E\left(G_{1} V, G_{2}\right)$ is non zero, then $G_{1} V$ and $G_{2}$ satisfy the following condition: The algebra $A$ is generated by elements $S q^{2^{i}}$. Let $h_{i}: A \longrightarrow \mathbb{Z}_{2}$ be the linear map which is zero on decomposables and $h_{i}\left(S q^{2^{j}}=\delta_{i, j}\right.$. Let $x,\left\{y_{i}\right\}$ and $z$ be $A$ generators of $H^{*}\left(K_{k}\right), H^{*}(T B \wedge$ $\left.K_{n+1}\right)$ and $H^{*}\left(K_{k+2 n+2}\right)$. Then $G_{2}^{*}(z)=\sum a_{i} y_{i}$, and $G_{1}^{*}\left(y_{i}\right)=b_{i} x$. (Since $G_{2} G_{1} V$ is homotopic to zero, $\sum a_{i} b_{i}=0$.)

Theorem 8.6 (Adams). If a map $S^{2 n+k} \longrightarrow S^{k} \longrightarrow E\left(G_{1} V, G_{2}\right)$ is non-zero, then for some $s$ and $t \leq s, \sum h_{s}\left(a_{i}\right) h_{t}\left(b_{i}\right)=0$.

Using the fact that $\chi\left(S q^{2^{i}}\right)=S q^{2^{i}}+$ decomposables, and inspecting the elements in 8.2 and 8.4, one sees that the condition in 8.6 is satisfied exactly when $n$ is of the form $2^{i}-1$. If a framed $2 n$-manifold has Kervaire invariant one, it will be non-zero in $\Omega_{2 n}\left(B O\left[v_{n+1}\right]\right)$. Conversely, if $h_{i}^{2}$ lives to $E_{\infty}$, there is a non-zero map $s: S^{2^{i}-2+k} \longrightarrow S^{k} \longrightarrow E\left(G_{1}, G_{2}\right)$. By an easy Hopf invariant one type argument, it goes to zero in $E_{G_{1}}$ and hence must be the $\pi_{*}$ generator of the fiber of $E\left(G_{1}, G_{2}\right) \longrightarrow E_{G_{1}}, K_{2^{i}-2+k}$. But this generator corresponds to $\left(S^{2^{i}-1} \times S^{2^{i}-1}, q\right)$ since by 7.6 this manifold has Kervaire invariant one and the underlying map of $q$ factors through $K_{n}$. Hence,

Corollary 8.7 (Browder). There is a framed $2 n$-manifold with Kervaire invariant one, if and only if $n=2^{i}-1$ and $h_{i}^{2}$ lives to $E_{\infty}$ in the Adams spectral sequence for $\pi_{*}\left(S^{0}\right)$.

\section{OTHER WORK}

An amusing low dimensional application of the generalized Kervaire invariant is afforded by immersions of surfaces $=$ closed, compact smooth 2-manifolds in $\mathbb{R}^{3}$. If $f: S \longrightarrow \mathbb{R}^{3}$ is such an immersion, associate to it $\phi: H_{1}(S)=H_{1}\left(S ; \mathbb{Z}_{2}\right) \longrightarrow \mathbb{Z}_{4}$ as follows. Represent $u \in H_{1}(S)$ by an embedded circle and let $T$ be a tubular neighborhood in $S$ of this circle. Define $\phi(u)$ to be the number of half twists (by $180^{\circ}$ ) of the twisted strip 
$f(T)$. This makes sense mod 4 and $\phi$ has the quadratic property with respect to the intersection pairing on $H_{1}(S)$. Then the quadratic functions associated to the intersection pairing are in one to one correspondence with the regular homotopy classes of immersion of $S$ in $\mathbb{R}^{3}$ and the Kervaire invariant gives an isomorphism of the cobordism group of such immersions onto $\mathbb{Z}_{8}([6])$.

Ochanine has generalized the above to surfaces immersed in 3-manifolds and to a $(8 a+2)$-manifold $V$ immersed in a $(8 a+4)$-manifold and dual to $w_{2}(M)$. He also related $K O$ characteristic classes for Spin $(8 a+2)$ manifolds to these issues $([18])$.

A variant of the above is to take $S$ with boundary $S^{1}$ and $f: S \longrightarrow \mathbb{R}^{3}$ an embedding. Then $f(\partial S)$ is a knot. In this connection the Kervaire invariant appears in a number of knot and link theory papers. For example, Levine expresses the Kervaire invariant of a knot in terms of its Alexander polynomial $([12])$.

There are a number of papers in homotopy theory studying the existence of framed manifolds having Kervaire invariant one, for example [13]. The existence of such manifolds in dimensions 30 and 62 was first proved by homotopy groups of spheres calculations ([2], [14]). In [8] Jones constructed a stably framed 30-manifold with Kervaire invariant one and also proved that a similar construction does not work in dimension 62. In [9] Browder's results for $2 n \neq 2^{j}-2$ are deduced from the Kahn-Priddy theorem.

\section{REFERENCES}

[1] J. F. Adams, The non-existence of elements of Hopf invariant one, Ann. of Math. (2) 72 (1960), 20-104.

[2] M. G. Barratt, J. D. S. Jones, and M. E. Mahowald, Relations amongst Toda brackets and the Kervaire invariant in dimension 62, J. London Math. Soc. (2) 30 (1984), no. 3, 533-550.

[3] W. Browder, The Kervaire invariant of framed manifolds and its generalization, Ann. of Math. (2) 90 (1969), 157-186.

[4] W. Browder, Surgery on simply connected manifolds, Springer-Verlag, New York-Heidelberg, 1972.

[5] E. H. Brown, Note on an invariant of Kervaire, Michigan Math. J. 12 (1965), 23-24.

[6] E. H. Brown, Generalizations of the Kervaire invariant, Ann. of Math. (2) 95 (1972), 368-383.

[7] E. H. Brown and F. P. Peterson, The Kervaire invariant of $(8 k+2)$ manifolds, Amer. J. Math. 88 (1966), 815-826.

[8] J. D. S. Jones, The Kervaire invariant of extended power manifolds, Topology 17 (1978), 249-266. 
[9] J. D. S. Jones and E. Rees, A note on the Kervaire invariant, Bull. London Math. Soc. 7 (1975) 279-282.

[10] M. A. Kervaire, A manifold that does not admit any differential structure, Comment. Math. Helv. 34 (1960), 257-270.

[11] M. A. Kervaire and J. W. Milnor, Groups of homotopy spheres I, Ann. of Math. (2) 77 (1963), 504-537.

[12] J. Levine, Polynomial invariants of knots of codimension two, Ann. of Math. (2) 83 (1966), 537-554.

[13] M. E. Mahowald, Some remarks on the Kervaire invariant problem from a homotopy point of view, Proc. Symp. Pure Math., Vol. XXII, Amer. Math. Soc., Providence, 1971.

[14] M. E. Mahowald and M. Tangora, Some differentials in the Adams spectral sequence, Pacif. J. Math. 60 (1975), 235-275.

[15] J. W. Milnor, A procedure for killing the homotopy groups of differentiable manifolds, Proc. Symposia in Pure Math., vol. III (1961), Amer. Math. Soc., Providence, pp. 39-55.

[16] S. P. Novikov, Homotopy equivalent manifolds I, Izv. Akad. Nauk SSSR, Ser. Mat. 28 (1964), 365-474.

[17] S. Ochanine, Signature modulo 16, invariants de Kervaire generalisés et nombres caractéristiques dans la $K$-théorie réelle, Mem. Soc. Math. France 81, no. 5 (1980).

[18] S. Ochanine, Formules "à la Hirzebruch" pour les invariants de Kervaire generalisés, C.R. Acad. Sci. Paris Ser. A-B 289 (1979), no. 9, 487-490.

[19] A. Ranicki, Exact sequences in the algebraic theory of surgery, Mathematical Notes 26, Princeton University Press, 1981.

[20] E. H. Spanier, Algebraic Topology, McGraw-Hill, 1966.

[21] R. Thom, Quelques propriétés globales des variétés différentiables, Com. Math. Helv. 28 (1954), 17-86.

[22] C. T. C. Wall, Surgery on compact manifolds, Academic Press, London and New York, 1970.

[23] M. Weiss, Surgery and the generalized Kervaire invariant, I, Proc. Lond. Math. Soc. 51 (1985), 146-192; II, ibid., 193-230.

[24] M. Weiss, Visible L-theory, Forum Math. 4 (1992), 465-498.

Department of Mathematics

BRANDEIS UNIVERSITY

WALTHAM, MA 02254-9110

E-mail address: brown@math.brandeis.edu 